
and Aromatic Plants

An International Journal ISSN: 2619-9645 | e-ISSN: 2667-5722

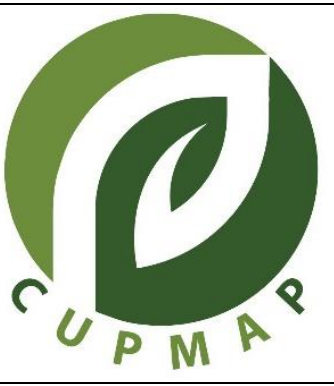

\title{
Characterization and Optimization of Phytosome Formulation Containing Alcohol-free Umckalin from Pelargonium sidoides
}

\author{
Ismail ASLAN ${ }^{1,2 *}$, (D) Ahmet Akif KURT ${ }^{3}$, \\ ${ }^{1}$ Department of Pharmacy Services, Hamidiye Vocational School of Health Services, University of Health \\ Sciences Turkey, 34668, Uskudar, Istanbul-Turkey \\ ${ }^{2}$ SFA R\&D Private Health Services Ltd Co.,Technopark Blv, No:1 3A/Z01, 34906, Pendik, İstanbul-Turkey \\ 3 Vocational School of Health Services, Suleyman Demirel University, 32200 Isparta-Turkey \\ *Corresponding author : eczismailaslan@gmail.com
}

\begin{abstract}
This research study was designed to investigate physicochemical properties of types of phytosome containing alcohol-free umckalin from Pelargonium sidoides. Fourteen different phytosomal formulations were prepared by film method using P. sidoides in various types of phosphatidylcholine; Phospholipon 85 G (P 85 G) and phospholipon 100H (PL 100H). Physicochemical properties were measured by particle size and size distribution, zeta potential and polydispersity index of optimum 4 phytosomal formulation. Polarized light microscopy (PLM) techniques were employed for obtaining size distribution and surface appearance and lamellarity. The determination of vesicle types of phytosomes was performed by using PLM. The most suitable alcohol-free phytosome combination was chosen as PL 100H: DCP: CHOL (10: 1: 4) containing $1 \%$ P.S (UP 4) because of its particle size and distribution $(<1000$ $\mathrm{nm})$ and zeta potential $(<-30 \mathrm{mV})$.
\end{abstract}

Key Words: Liposome, Phytosome, Umckalin, Pelargonium sidoides, Alcohol-free extract.

(c) CUPMAP. All rights reserved.

\section{Introduction}

Plants have been traditionally used against variety diseases since ancient times (Aslan, 2007). Pelargonium sidoides has an ethnobotanical and traditional plant in the treatment of some of the specific problems such as acute bronchitis, tonsillitis, pharyngitis, sinusitis and symptoms of the common flu diseases since from $20^{\text {th }}$ century (Brendler and Van Wky, 2008; Lizogub et al., 2007).

The underground parts of Pelargonium sidoides are very well-known to include a rich of quite oxygenated coumarins and some other phenolic and polyphenolic substituents (Sharma and Yadav 2017). Pelargonium sidoides showed in vitro bactericidal, virucidal, and immune system regulating characteristics in many scientific researches (Kolodziej, 2011; Kolodziej and Kiderlen, 2007).

Umckalin is one of the important compound of the Pelargonium sidoides (P.S.) collected from its root via alcoholic extraction method. It has been widely used against common cold diseases in children (Lalli et al., 2008). 
However, these extracts may cause problem for children and addictive patient especially in syrup formulations because of alcohol comtent (Agnich et al., 2013).

Phytosomes are submicron spheres composed of membrane similar phospholipid layers surrounding aqueous compartment including phytoactive molecules (Kidd and Head, 2005). The lipid structure is accomplished basically of phospholipid components. Phospholipids are amphiphilic; they have a hydrophilic head and a lipophilic tail. In aqueous solutions, they are arranged in bilayers, which form closed vesicles like artificial cells (Duman et al., 2014).

P.S. extract containing umckalin includes approximately $11 \%$ ethanol in traditional and medicinal products (Wopker et al., 2020). However, ethanol containing products are reported as hazardous for infants and children (Batista et al., 2020). The extraction with the solvent mixture containing ethanol free was defined, thus obtaining a liquid preparation that provides a convenient use for children (Kohnen, 2007).
Here we present the detailed evaluation of the physicochemical properties of phytosome containing alcohol free Pelargonium sidoides in particle size distribution, zeta potential, polydispersity index and polarized light image parameters. The purpose of this research is to provide an optimum formulation of liposome combinations. This paper will focus mainly on the primer and seconder stability characteristics of phytosome formulations.

\section{Material and Methods}

\subsection{Materials}

Phospholipon $100 \quad \mathrm{H} \quad(\mathrm{P} 100 \mathrm{H})$ and Phospholipon 85 G (P85G) were provided from LIPOID GmbH (Germany). Stearylamine (SA), Dicetyl phosphate (DCP), Cholesterol (CHOL) were purchased from Sigma (UK). Pelargonium sidoides containing umckalin product (Umka®) was provided from Abdi İbrahim Pharmaceuticals. All other chemicals were of analytical grade. Polarized Light Microscope (PLM) was used in order to determine the vesicle types (NIKON).

Table 1. Phytosome formulations and their compositions.

\begin{tabular}{llllll}
\hline Code & Composition & pH & $\begin{array}{l}\text { Molar } \\
\text { Ratio }\end{array}$ & Observation & Result \\
\hline UPF-1 & PL85G:CHOL + 1\% P.S. & 5.5 & $3: 2$ & Phase separation & Eliminated \\
UPF-2 & PL85G:CHOL + 1\% P.S. & 5.5 & $7: 2$ & Phase separation & Eliminated \\
UPF-3 & PL85G:CHOL + 1\% P.S. & 5.5 & $10: 4$ & Phase separation & Eliminated \\
UPF-4 & PL100H:CHOL+ 1\% P.S. & 5.5 & $3: 2$ & Phase separation & Eliminated \\
UPF-5 & PL100H:CHOL+ 1\% P.S. & 5.5 & $7: 2$ & Phase separation & Eliminated \\
UPF-6 & PL100H:CHOL+ 1\% P.S. & 5.5 & $10: 4$ & Phase separation & Eliminated \\
UPF-7 & PL85G:SA:CHOL+ 1\% P.S. & 5.5 & $3: 1: 2$ & Heterogenous & Eliminated \\
UPF-8 & PL100H:SA:CHOL+ 1\% P.S. & 5.5 & $3: 1: 2$ & Heterogenous & Eliminated \\
UPF-9 & PL85G:DCP:CHOL+ 1\% P.S. & 5.5 & $3: 1: 2$ & Heterogenous & Eliminated \\
UPF-10 & PL100H:DCP:CHOL+ 1\% P.S. & 5.5 & $3: 1: 2$ & Heterogenous & Eliminated \\
UP-1 & PL85G:SA:CHOL+ 1\% P.S. & $\mathbf{5 . 5}$ & $\mathbf{7 : 1 : 2}$ & Milky & Selected \\
UP-2 & PL100H:SA:CHOL+ 1\% P.S. & $\mathbf{5 . 5}$ & $\mathbf{7 : 1 : 2}$ & Milky & Selected \\
UP-3 & PL85G:DCP:CHOL+ 1\% P.S. & $\mathbf{5 . 5}$ & $\mathbf{1 0 : 1 : 4}$ & Milky & Selected \\
UP-4 & PL100H:DCP:CHOL+1\%P.S. & $\mathbf{5 . 5}$ & $\mathbf{1 0 : 1 : 4}$ & Milky & Selected \\
\hline
\end{tabular}




\subsection{Phytosome Formulations and Preparation Technique}

Phytosome dispersions were prepared by film technique (Gunal et al., 2019). Briefly, phytosome was prepared by dissolving the $40 \mu \mathrm{mol} \mathrm{mL}-1$ of phospholipids in $30 \mathrm{~mL}$ chloroform in a round-bottom flask. Organic solvent was removed using a rotary evaporator (Heidolph, Germany) under reduced pressure to form a thin film over the wall of the flask. In addition, ethanol was accurately evaporated using rotary evaporator from plant extract. The dried film was then hydrated over a water bath with buffer alcohol free P.S. containing umckalin. Fourteen different formulations and details are represented in Table 1.

\subsection{Characterization of Phytosome Formulations}

Selected four phytosome formulations were characterized by mean particle size and size distribution, zeta potential and polydispersity index. $0.1 \mathrm{ml}$ of liposomes dispersions were diluted with $0.9 \mathrm{ml}$ of 10 $\mathrm{mM}$ phosphate ( $\mathrm{pH}$ 5.5) buffer after phytosome preparation. The size distributions of the phytosome were measured by dynamic light scattering (DLS) using Particle Sizer (Malvern, UK). The mean particle size and size distribution and polydispersity index results were obtained as

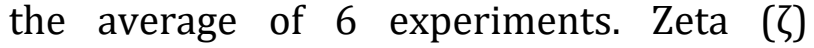
potential was measured by using a Zetasizer and each result was the mean of 10 measurements. All the measurements were performed at $25^{\circ} \mathrm{C}$ and at an angle of $90^{\circ}$.
The determination of vesicle types of phytosome was performed by using Polarized light microscope (PLM). Magnification of the microscope was $100 \mathrm{X}$ the each phytosome dispersion. PLM techniques were employed for obtaining size distribution and surface appearance and lamellarity for 4 optimum formulation.

\section{Results and Discussion}

\subsection{Characterization Results}

Particle size is an essential factor of bioavailability for drug delivery systems such as phytosomes. According to particle size distribution results, all formulations were found below $1000 \mathrm{~nm}$ average diameter without UP3 as $676 \mathrm{~nm} \pm 5.73,908 \mathrm{~nm} \pm$ 24.40, $1146 \mathrm{~nm} \pm 12.30,674 \mathrm{~nm} \pm 30.48$, respectively.

The zeta potential is a significant property of the cumulative charge of a particle, and differences in size reflect sedimentation or fusion. The most stable phytosomes are around $30 \mathrm{mV}$. In this research paper, zeta potential results were found as $23.1 \mathrm{mV} \pm$ $1.04,42.9 \mathrm{mV} \pm 1.12,-49.7 \pm 1.11,-43.7 \pm$ 1.03 , respectively. The maximum stability and the minimum aggregation occur at that zeta potential value $(30-50 \mathrm{mV})$ so that expected from DLVO theory. When the zeta potential goes below the critical value $(<30$ $\mathrm{mV}$ ), the attractive forces supersede the repulsive forces and flocculation occurs. These loosely packed particles or flocs settle faster than the deflocculated particles because of their larger sizes (Awad et al., 2005; Kumar et al., 2007).

Table 2. Results of particle size, zeta potential and polydispersity index of phytosomes $(n=3)$.

\begin{tabular}{llllll}
\hline Code & Composition & $\begin{array}{l}\text { Molar } \\
\text { Ratio }\end{array}$ & $\begin{array}{l}\text { Average } \\
\text { diameter (nm) }\end{array}$ & PDI & $\begin{array}{l}\text { Zeta } \\
\text { potential (mV) }\end{array}$ \\
\hline UP-1 & PL85G:SA:CHOL+ 1\% P.S. & $7: 1: 2$ & $676 \pm 5.73$ & $0.461 \pm 0.02$ & $+23.1 \pm 1.04$ \\
UP-2 & PL100H:SA:CHOL+ 1\% P.S. & $7: 1: 2$ & $908 \pm 24.40$ & $0.670 \pm 0.03$ & $+42.9 \pm 1.12$ \\
UP-3 & PL85G:DCP:CHOL+ 1\% P.S. & $10: 1: 4$ & $1146 \pm 12.30$ & $0.568 \pm 0.02$ & $-49.7 \pm 1.11$ \\
UP-4 & PL100H:DCP:CHOL+1\%P.S. & $10: 1: 4$ & $674 \pm 30.48$ & $0.323 \pm 0.01$ & $-43.7 \pm 1.03$ \\
\hline
\end{tabular}


When the results are evaluated from PDI (poly dispersity index) viewpoint, it was determined PDI as $0.461 \pm 0.02,0.670 \pm 0.03$, $0.568 \pm 0.02,0.323 \pm 0.01$, respectively. In addition, it was reported that uniform and stable phytosome dispersions have $<0.5$ PDI value (Colas et al., 2007). In this research, PDI value was found as $0.323 \pm 0.01 \mathrm{~nm}$ optimum for UP4. All data were given Table 2.

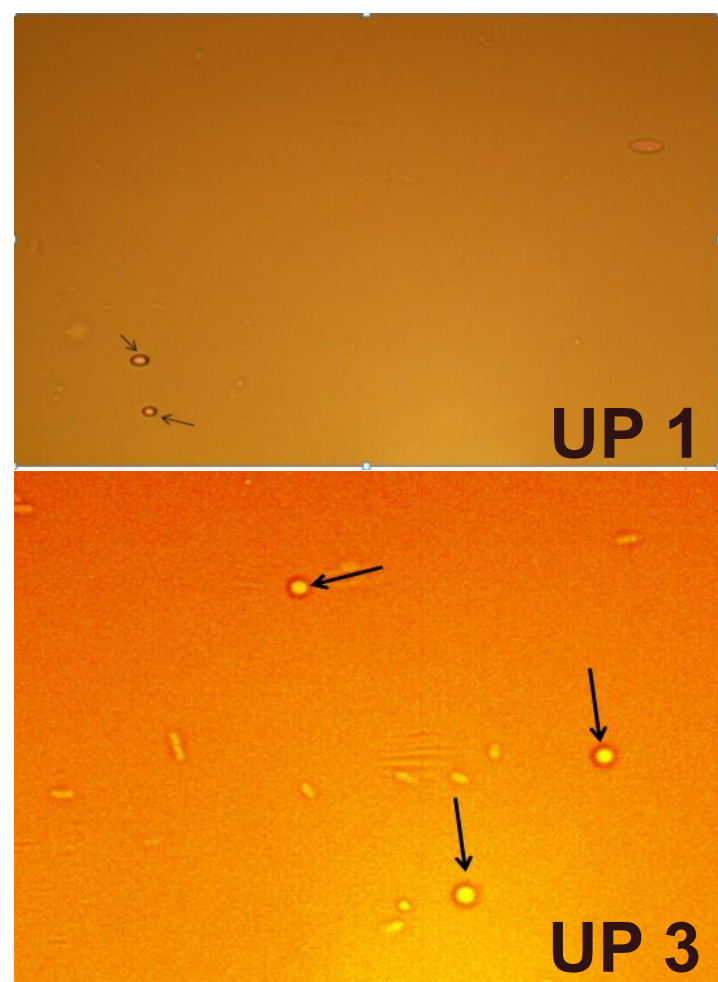

Figure 1. PLM images of phytosome formulations (UP1, UP2, UP3, UP4).

\section{Conclusion}

In this study. appearance, mean of particle size and size distribution, polydispersity index, and zeta potential values were considered to select the optimum phytosome formulation. So, the optimum formulation was selected depending on the formulations from UP1 to UP4 (Table1). Two types of phytosome formulations were designed (gel and liquid). Phase transition temperature of gel state liposomes (PL100H) was higher than liquid state liposomes (P85G). Stearylamine and dicetylphosphate were employed as charge inducer negative and positive, respectively.

\subsection{Microscopic Observation Results}

Microscopic observations of the liposomes were imaged with Polarized Light Microscope. According to PLM results, multilamellar vesicle type liposomes were mainly observed in all formulations in Figure 1.

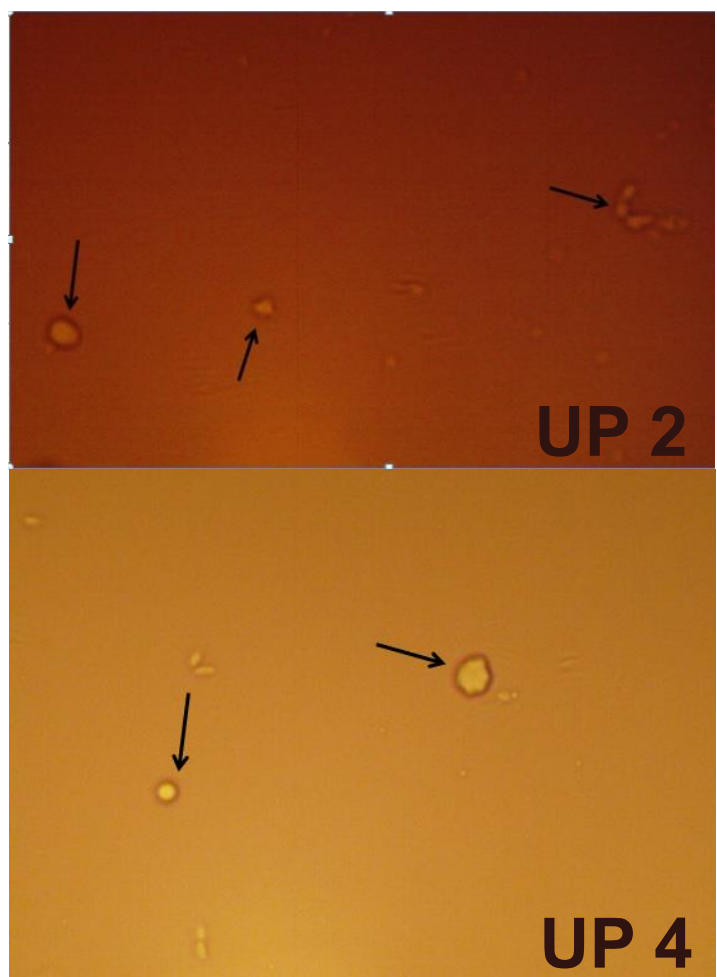

The charge inducer was incorporated into the liposome bilayer in order to stabilize liposome dispersions physically. This effect depends on electrostatic interaction between vesicles and it provides electrostatic charge to the vesicle surface (Ethemoglu et al., 2017). The objective of this study is to develop a delivery system, which is containing alcohol free P.S. for children, with novel carrier system and stable phytosome formulation. The optimum liposome formulation (UP4) composed of (PL 100H: SA: CHOL - 1\% P.S.) was selected in all formulations. 


\section{Acknowledgements}

The authors would like to special thanks to MSc. Cosm. Bekir Çakıcı and MSc Biologist Leyla Tarhan (SFA R\&D Laboratories, İstanbul, Turkey) for their valuable contribution to our research article.

\section{Conflict of Interest}

The authors declare that they have no conflict of interest.

\section{References}

1. Agnich, L. E., Stogner, J. M., Miller, B. L., Marcum, C. D. (2013). Purple drank prevalence and characteristics of misusers of codeine cough syrup mixtures. Addictive behaviors, 38(9), 2445-2449. https://doi.org/10.1016/j.addbeh.2013.03.020.

2. Aslan, I. (2007) Plants and Cosmetics. Fitomed, 3, 4951.

3. Awad, D., Tabod, I., Lutz, S., Wessolowski, H., Gabel, D. (2005). Interaction of Na2B12H11SH with liposomes: Influence on zeta potential and particle size. Journal of organometallic chemistry, 690(11), 2732-2735.

https://doi.org/10.1016/i.jorganchem.2005.01.013.

4. Batista, Lilian R., Antoniosi Filho, Nelson R.. (2020). Ethanol Content Determination in Medicine Syrups Using Headspace and Multidimensional Heart-Cut Gas Chromatography Coupled to Mass Spectrometry. Journal of the Brazilian Chemical Society, 31(2), 394401. Epub January 20, 2020. https://doi.org/10.21577/01035053.20190193.

5. Brendler T, van Wyk BE. A historical, scientific and commercial perspective on the medicinal use of Pelargonium sidoides (Geraniaceae). J Ethnopharmacol.b 2008;119(3):420-433. https://doi.org/10.1016/i.jep.2008.07.037.

6. Colas, J. C., Shi, W., Rao, V. S., Omri, A., Mozafari, M. R., Singh, H. (2007). Microscopical investigations of nisin-loaded nanoliposomes prepared by Mozafari method and their bacterial targeting. Micron (Oxford, England : 1993), 38(8), 841-847. https://doi.org/10.1016/j.micron.2007.06.013.

7. Duman, G., Aslan, İ., Özer, A. Y., İnanç, İ., Taralp, A. (2014). Liposome, gel and lipogelosome formulations containing sodium hyaluronate. Journal of liposome research, 24(4), 259-269. https://doi.org/10.3109/08982104.2014.907305.

8. Ethemoglu, M. S., Seker, F. B., Akkaya, H., Kilic, E., Aslan, I., Erdogan, C. S., Yilmaz, B. (2017). Anticonvulsant activity of resveratrol-loaded liposomes in vivo. Neuroscience, 357, 12-19. https://doi.org/10.1016/j.neuroscience.2017.05.02 6.
9. Gunal, M. Y., Ayla, Ş., Bedri, N., Beker, M. C.., Cağlayan, A. B., Aslan, İ. ... Kılıç, Ü. (2019). The effects of topical liposomal resveratrol on incisional and excisional wound healing process. Turkderm-Turkish Archives of Dermatology and Venerology, 53(4), 128-134. http://doi.org/10.4274/turkderm.galenos.2019.82 612.

10. Kidd, P., \& Head, K. (2005). A review of the bioavailability and clinical efficacy of milk thistle phytosome: a silybin-phosphatidylcholine complex (Siliphos). Alternative medicine review : a journal of clinical therapeutic, 10(3), 193-203. http://europepmc.org/abstract/MED/16164374.

11. Kohnen, W. (2007). Method for extracting plants of the genus Pelargonium sidoides extract produced according to said method, and use thereof. World PatentW02007009446.

12. Kolodziej H. (2011). Antimicrobial, Antiviral and Immunomodulatory Activity Studies of Pelargonium sidoides (EPs ${ }^{\circledR}$ 7630) in the Context of Health Promotion. Pharmaceuticals (Basel, Switzerland), 4(10), 1295-1314. https://doi.org/10.3390/ph4101295.

13. Kolodziej, H., Kiderlen, A. F. (2007). In vitro evaluation of antibacterial and immunomodulatory activities of Pelargonium reniforme, Pelargonium sidoides and the related herbal drug preparation EPs 7630. Phytomedicine, 14 Suppl 6, 18-26. https://doi.org/10.1016/i.phymed.2006.11.020.

14. Kumar, R., Singh, B., Bakshi, G., \& Katare, O. P. (2007). Development of liposomal systems of finasteride for topical applications: design, characterization, and in vitro evaluation. Pharmaceutical development and technology, 12(6),591-601. https://doi.org/10.1080/10837450701481181.

15. Lalli, J. Y. Y., Van Zyl, R. L., Van Vuuren, S. F., Viljoen, A. M. (2008). In vitro biological activities of South African Pelargonium (Geraniaceae) species. South African Journal of Botany, 74(1), 153-157. https://doi.org/10.1016/j.sajb.2007.08.011.

16. Lizogub VG, Riley DS, Heger M. Efficacy of a pelargonium sidoides preparation in patients with the common cold: a randomized, double blind, placebo-controlled clinical trial. Explore (New York, N.Y.). $2007 \quad$ Nov-Dec;3(6):573-584. https://doi.org/10.1016/i.explore.2007.09.004.

17. Sharma D, Yadav JP. An Overview of Phytotherapeutic Approaches for the Treatment of Tuberculosis. Mini Rev Med Chem. 2017;17(2):167183.

https://doi.org/10.2174/13895575166661605051 14603.

18. Wopker, P. M., Schwermer, M., Sommer, S., Längler, A., Fetz, K., Ostermann, T., Zuzak, T. J. (2020). Complementary and alternative medicine in the treatment of acute bronchitis in children: A systematic review. Complementary therapies in medicine, 49 102217 https://doi.org/10.1016/i.ctim.2019.102217. 